\title{
Absence of singular continuous spectrum for some geometric Laplacians
}

\author{
Leonardo A. Cano García
}

September 13, 2018

\begin{abstract}
We provide two examples of spectral analysis techniques of Schrödinger operators applied to geometric Laplacians. In particular we show how to adapt the method of analytic dilation to Laplacians on complete manifolds with corners of codimension 2 finding the absence of singular continuous spectrum for these operators, a description of the behavior of its pure point spectrum in terms of the underlying geometry, and a theory of quantum resonances.
\end{abstract}

\section{Introduction}

Spectral geometry studies the interactions between the geometry of a Riemannian manifold and the spectral analysis of its associated Laplacian. These interactions have been deeply studied in the case in which the manifold is closed (see [Cha84] [Ros97]). In the closed case the Laplacian is a self-adjoint operator with compact resolvent and hence its spectrum is purely discrete. This contrasts with the general case since, if $(M, g)$ is a geodesically complete Riemannian manifold, not necessarily closed, it is known that its Laplacian $\Delta_{g}: C_{c}^{\infty}(M) \rightarrow L^{2}\left(M, d v o l_{g}\right)$ is essentially self-adjoint but its spectral resolution is not purely discrete in general.

In order to show more clearly the new spectral phenomenons for $\Delta_{g}$ that the lost of compactness of $M$ brings with, let us remind the spectral theorem for self-adjoint operators.

Theorem 1 [RS80] Let $A$ be a self-adjoint operator acting on a separable Hilbert space $\mathscr{H}$. Then, there exists a collection of measures on $\mathbb{R},\left\{\mu_{i}\right\}_{i \in I}$ where $I \subseteq \mathbb{N}$, and there 
exists a unitary operator $\mathscr{F}$ such that the following diagram commutes

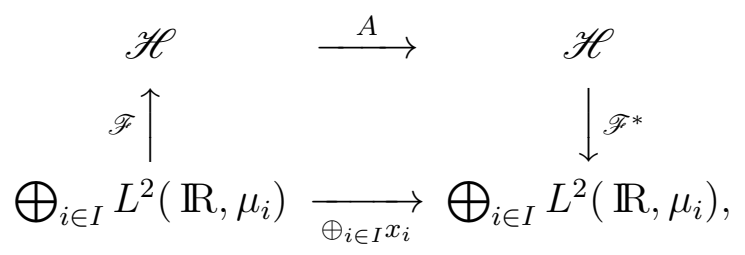

where the operator $\oplus_{i \in I} x_{i}$ is the direct sum of the multiplication operators $x_{i}: L^{2}\left(\mathbb{R}, \mu_{i}\right) \rightarrow$ $L^{2}\left(\mathbb{R}, \mu_{i}\right)$ that, specifically, send a function $f \in L^{2}\left(\mathbb{R}, \mu_{i}\right)$ to the function $x_{i} f$.

Given a self-adjoint operator $A$, as in the theorem above, the Lebesgue decomposition theorem for measures induces a decomposition of the Hilbert space $\mathscr{H}$ into three important invariants subspaces $\mathscr{H}_{a c}, \mathscr{H}_{p p}$ and $\mathscr{H}_{\text {sing }}$ that, using theorem 1, are the associated to the three Hilbert spaces

$$
\bigoplus_{\mu_{i} \text { is ac }} L^{2}\left(\mathbb{R}, \mu_{i}\right), \bigoplus_{\mu_{i} \text { is pp }} L^{2}\left(\mathbb{R}, \mu_{i}\right) \text { and } \bigoplus_{\mu_{i} \text { is s }} L^{2}\left(\mathbb{R}, \mu_{i}\right)
$$

where ac means absolutely continuous, pp means pure point and s means singular continuous measures (all of them defined respect to the Lebesgue measure in $\mathbb{R}$ ). We have already said that for $(M, g)$ a closed Riemannian manifold, its Laplacian has purely discrete spectrum, in other words the invariant subspaces $L_{a c}^{2}\left(M, d v o l_{g}\right)$ and $L_{\text {sing }}^{2}\left(M, d v o l_{g}\right)$, corresponding to the self-adjoint operator $A:=\Delta_{g}$, are empty. In this paper we will show examples of manifolds $(M, g)$ for which $L_{a c}^{2}\left(M, d v o l_{g}\right)$ is not empty and $L_{\text {sing }}^{2}\left(M, d v o l_{g}\right)$ is empty.

The former description of the spectral analysis of self-adjoint operators justifies the question about how to prove that a geometric Laplacian does not have singular continuous spectrum. This paper provide, through the friendly environment of precise examples and without pretending to give a complete answer, an illustration of how to work around this question. The common feature of these examples is the control on the Riemannian metric of the open manifold at infinity.

Let us describe more carefully the contents. For Laplacians acting on manifolds with cylindrical or cusps ends and their natural generalizations it is well known in the literature (see Gui89] Hus05] Mül83]) how to find a meromorphic extension of their resolvent and how, using it, to prove the absence of singular continuous spectrum. In section 1 we illustrate the classical method to obtain a meromorphic extension of the resolvent for manifolds with cylindrical and cusp ends, and we show the relation of it with the absence of singular continuous spectrum, that is, fundamentally, the limit absorption principle. In section 2 we explain the notion of complete manifold with 
corner of codimension 2 and how to prove absence of singular continuous spectrum for Laplacians on this kind of manifolds using the method of analylic dilation. Section 2 describe following Can11] the way to apply the technique of analytic dilation to Laplacians on complete manifolds with corners of codimension 2, context on which the techniques explained in section 1 do not apply. The method of analytic dilation was originally applied to $N$-particle Schrödinger operators, a classic reference in that setting is Gér93. It has also been applied to the black-box perturbations of the Euclidean Laplacian in the series of papers [SZ93b] SZ93a] SZ94] SZ295]. In [Bal97] it is used to study Laplacians on hyperbolic manifolds. The analytic dilation has also been applied to the study of the spectral and scattering theory of quantum wave guides and Dirichlet boundary domains, see e.g. [DEM98] [KS07]. It has also been applied to arbitrary symmetric spaces of noncompact type in [MV02 [MV04 [MV07. In [Kal10] it is applied to manifolds with analytic asimptotically cylindrical end. In each of these settings new ideas and new methods carry out.

\section{Meromorphic extension of the resolvent and sin- gular continuous spectrum}

In this section we give the main ideas of a method for meromorphically extend the resolvent of Laplacians on manifolds with cylindrical and cusps ends and show why such extension is enough to have absence of singular continuous spectrum. The results of this section were obtained in Gui89 [Mül83] but we base our exposition in [Hus05] since we consider it is easier to understand for non experts.

Definition 1 Let $M$ be an open manifold with a decomposition in a compact manifold $M_{0}$ with boundary $Y:=\partial M_{0}$ and an open manifold $M_{\infty}$ with boundary and suppose that $\partial M_{\infty}=M_{0} \cap M_{\infty}=\partial M_{0}$. If $M$ is endowed with a complete Riemannian metric such that

i) $M_{\infty}$ is isometric to $Y \times \mathbb{R}_{+}$with the natural product metric $g_{y}+d u \otimes d u$, we say that $M$ is a manifold with cylindrical end.

ii) $M_{\infty}$ is isometric to $Y \times \mathbb{R}_{+}$with the Riemannian metric $\frac{d u \otimes d u}{u^{2}}+\frac{g_{y}}{u^{2}}$, we say that $M$ is a a manifold with cusp end. 
Let $\Delta_{c y l}$ be the Laplacian associated to a manifold $\left(M, g_{M}\right)$ with cylindrical end. We recall that on $M_{\infty}=Y \times \mathbb{R}_{+}$the Laplacian $\Delta_{c y l}$ has the form

$$
\Delta_{0, M}:=-\frac{\partial^{2}}{\partial u^{2}}+\Delta_{Y}
$$

where $\Delta_{Y}$ is the Laplacian on $Y$ associated to the Riemannian metric $g_{Y}$. Let $\left(N^{n}, g_{N}\right)$ be a manifold with cusp, with $N_{\infty}=Y \times \mathbb{R}_{+}$, and let $\Delta_{\text {cusp }}$ be the Laplacian associated to $\left(N^{n}, g_{N}\right) . \Delta_{\text {cusp }}$ has the form

$$
\Delta_{0, N}:=-u^{2} \frac{\partial^{2}}{\partial u^{2}}+(n-1) u \frac{\partial}{\partial u}+u^{2} \Delta_{Y}
$$

on $N_{\infty}$. An important approach, in quantum scattering theory, is to think that the Laplacians $\Delta_{c y l}$ and $\Delta_{c u s p}$ are perturbations of the operators $\Delta_{0, M}$ and $\Delta_{0, N}$ respectively. Roughly speaking, this idea is formalized in the following way. Using Dirichlet boundary conditions, we consider the operators $\Delta_{0, M}$ and $\Delta_{0, N}$ as self-adjoint operators in $L^{2}\left(Y \times \mathbb{R}_{+}, d v o l_{g_{M}}\right)$ and $L^{2}\left(Y \times \mathbb{R}_{+}, d v o l_{g_{N}}\right)$. Observe that $L^{2}\left(Y \times \mathbb{R}_{+}, d v o l_{g_{M}}\right)$ is naturally isomorphic to $L^{2}\left(Y, d v o l_{g_{Y}}\right) \otimes L^{2}\left(\mathbb{R}_{+}, d u\right)$; furthermore, if $\phi_{i} \in C^{\infty}(Y)$ is an orthonormal basis of eigenvectors of $\Delta_{Y}$ with eigenvalues $\mu_{i}$, then it provides an isomorphism of $L^{2}\left(Y, d v o l_{g_{Y}}\right) \otimes L^{2}\left(\mathbb{R}_{+}, d u\right)$ with $\oplus_{i \in \mathbb{N}} L^{2}\left(\mathbb{R}_{+}, d u\right)$, hence $L^{2}(Y \times$ $\left.\mathbb{R}_{+}, d v o l_{g_{M}}\right) \cong \oplus_{i \in \mathbb{N}} L^{2}\left(\mathbb{R}_{+}, d u\right)$. Modulo this last isomorphism, we have:

$$
\Delta_{0, M}=\bigoplus_{i \in \mathbb{N}}\left(-\frac{\partial^{2}}{\partial u^{2}}+\mu_{i}\right)
$$

The analogue of (11) in the context of manifolds with cusps is

$$
\Delta_{0, M}=\bigoplus_{i \in \mathbb{N}}\left(-u^{2} \frac{\partial^{2}}{\partial u^{2}}+(n-1) u \frac{\partial}{\partial u}+u^{2} \mu_{j}\right)
$$

From this point we continue our exposition over the manifold with cylindrical end $M$, indicating how analogous methods apply in the case of manifolds with cusps and refering to [M̈̈l83] for details.

Fourier transform and (2) give us a formula for the resolvent of the operators $-\frac{\partial^{2}}{\partial u^{2}}+$ $\mu_{i}$ and hence for the resolvent of $\Delta_{0, M}$. The analogue of such a formula in the case of the manifold $N$ with cusp is technically harder to obtain and can be found in [Mül83, lemma 2.68]. Define the double of the compact manifold with boundary $M_{0}$ as $\tilde{M}:=M_{0} \cup_{Y} M_{0}$, where we are identifying the boundary $Y$ of two disjoint copies of $M_{0}$ and we endow $\tilde{M}$ with the natural differential and Riemmanian structure. We have also a nice formula 
for the resolvent of the Laplacian $\Delta_{\tilde{M}}$ of $\tilde{M}$ using a spectral resolution of $\Delta_{\tilde{M}}$. In order to apply this knowledge about the resolvents in $M_{\infty}$ and $\tilde{M}$, we construct a parametrix for the resolvent of $\Delta_{M}$ i. e. an operator $P(\lambda)$ such that $R(\lambda)-P(\lambda)$ is compact in some weighted $L^{2}$-space, where $\lambda \in \mathbb{C}-\mathbb{R}_{+}$and $R(\lambda):=\left(\Delta_{M}-\lambda\right)^{-1}$ denotes the resolvent of $\Delta_{M}$. We proceed as in [Hus05]: for $0 \geq a \geq b$, let $\rho(a, b) \in C^{\infty}(M,[0,1])$ be such that

$$
\rho(a, b)(x)= \begin{cases}0 & \text { for } x \in M \cup(Y \times[0, a]) ; \\ 1 & \text { for } x \in Y \times[b, \infty) .\end{cases}
$$

We define the functions:

$$
\begin{array}{ll}
\Phi_{1}:=1-\rho\left(\frac{4}{5}, 1\right), & \Psi_{1}:=1-\rho\left(\frac{2}{5}, \frac{3}{5}\right) \\
\Phi_{2}:=\rho\left(0, \frac{1}{5}\right), & \Psi_{2}:=1-\Psi_{1},
\end{array}
$$

for which $\Psi_{1}+\Psi_{2}=1$,

$$
\Phi_{j}(x)=1 \text { for } x \in \operatorname{supp}\left(\Psi_{j}\right), \text { and } \operatorname{dist}\left(\operatorname{supp} \nabla \Phi_{j}, \operatorname{supp} \Psi_{j}\right) \geq \frac{1}{5}
$$

We define the operator $S(\lambda)$ with Schwartz kernel

$$
S\left(x_{1}, x_{2}, \lambda\right):=\sum_{j=1}^{2} \Psi_{j}\left(x_{1}\right) R_{j}\left(x_{1}, x_{2}, \lambda\right) \Phi_{j}\left(x_{2}\right)
$$

where $R_{1}\left(x_{1}, x_{2}, \lambda\right)$ is the Schwartz kernel of the resolvent of the Laplacian $\Delta_{\tilde{M}}$, on $\tilde{M}$ the double of the manifold with boundary $M_{0}$, and $R_{2}\left(x_{1}, x_{2}, \lambda\right)$ is the Schwartz kernel of the resolvent of $\Delta_{0, M}$. Using the explicit expressions of the Schwartz kernels $R_{1}\left(x_{1}, x_{2}, \lambda\right)$ and $R_{2}\left(x_{1}, x_{2}, \lambda\right)$ and (1) , it is possible to prove

Lemma 1 Hus05, lemma 3.8] For $\lambda \in \mathbb{C}-\mathbb{R}_{+}$, the operator $S(\lambda)$ is a parametrix of $R(\lambda)$ in the sense that $R(\lambda)-S(\lambda)$ is $L^{2}$-compact.

The meromorphic extension of the resolvent will have a domain contained in the following surface, that we call spectral surface:

$$
\Sigma_{s}:=\left\{\Lambda:=\left(\Lambda_{i}\right) \in \mathbb{C}^{\mathbb{N}}: \forall i, j \in \mathbb{N}, \Lambda_{i}^{2}+\mu_{i}=\Lambda_{j}^{2}+\mu_{j}\right\}
$$

$\Sigma_{s}$ is a covering of $\mathbb{C}$ with projection $\pi_{s}(\Lambda):=\Lambda_{i}^{2}+\mu_{i}$. 
As we said previously, we will consider the resolvent acting on weighted $L^{2}$-spaces that we shall define now:

$$
L_{\delta}^{2}(M):=\left\{f: M \stackrel{\text { meas }}{\rightarrow} \mathbb{C}: \int_{0}^{\infty} \int_{Y} e^{2 \delta u}|f(y, u)|^{2} d v o l_{g_{Y}} d u<\infty\right\} .
$$

For all $\delta>0$, we have the inclusions

$$
L_{\delta}^{2}(M) \subset L^{2}(M) \subset L_{-\delta}^{2}(M)
$$

If we define the physical domain FD by

$$
F D:=\left\{\Lambda \in \Sigma_{s}: \Lambda_{i} \geq 0\right\}
$$

then we can identify $F D$ with $\mathbb{C}-\mathbb{R}_{+}$. We denote by $\Sigma_{s}^{\mu}$ the connected component of $\pi_{s}^{-1}(\mathbb{C}-[\mu, \infty))$ and, for $\epsilon>0, \mu(\epsilon):=\min \left\{\mu \in \sigma\left(\Delta_{Y}\right): \mu \leq \epsilon\right\}$. Now we can define the domains $\Omega_{\epsilon}$, for $\epsilon>0$, where we will extend the resolvent of $\Delta_{M}$,

$$
\Omega_{\epsilon}:=\left(F D \cup \pi_{s}^{-1}(\{z \in \mathbb{C}:|z| \leq \epsilon\})\right) \cap \Sigma_{s}^{\mu(\epsilon)}
$$

From the explicit formulas of the resolvents $R_{1}(\lambda)$ and $R_{2}(\lambda)$ in equation (3)), and the definition of the weigthed $L^{2}$-spaces in (41), we deduce the following.

Lemma 2 [Hus05, lemma 3.20] For all $\delta \geq \epsilon>0$ the function $\lambda \mapsto S(\lambda)$ has a meromorphic extension to $\Omega_{\epsilon}$ as a continuous operator from $L_{\delta}^{2}(M)$ to $L_{-\delta}^{2}(M)$.

For $\Lambda \in \Omega_{\epsilon}$, let us define the operators

$$
G(\Lambda):=S(\Lambda)\left(\Delta_{M}-\pi_{s}(\Lambda)\right)-I d
$$

We will use the following important tool of functional analysis to meromorphically extend the resolvent.

Theorem 2 [Hus05, appendix] (Analytic Fredholm theorem) Let $U \subset \mathbb{C}$ be an open and connected set and let $T(z)$, for $z \in U$, be an analytic family of compact operators of a Hilbert space $\mathscr{H}$. Suppose that, for $z_{0} \in U,\left(I d-T\left(z_{0}\right)\right)^{-1}$ exists, then the family $(I d-T(z))^{-1}$ is meromorphic in $U$ with values in the bounded linear operators of $\mathscr{H}$ and poles contained in the set $\{z \in U: 1 \in \sigma(T(z))\}$.

As for lemma 1, it is possible to prove that $G(\Lambda)$, as a bounded operator of $L_{-\delta}^{2}(M)$, is compact. This fact, lemma 2 and theorem 2 imply 
Theorem 3 [Hus05, theorem 3.24] The resolvent $R(\lambda)$ has a meromorphic extension from $F D$ to $\Omega_{\epsilon}$ as a continuous operator from $L_{\delta}^{2}(M)$ to $L_{-\delta}^{2}(M)$.

The next theorem provides the connection between the meromorphic extension of the resolvent and the absence of singular continuous spectrum.

Theorem 4 [RS79] Let $H$ be a self-adjoint operator with resolvent $R(\lambda):=(H-\lambda)^{-1}$.

i) Let $(a, b)$ be a bounded interval and $\varphi \in \mathscr{H}$. Suppose that there exists $p>1$ for which:

$$
\sup _{0<\epsilon<1} \int_{a}^{b}|\operatorname{Im}(\varphi, R(x+i \epsilon) \varphi)|^{p} d x<\infty .
$$

Then $E_{(a, b)} \varphi \in \mathscr{H}_{a c}$.

ii) Let $(a, b)$ be a bounded interval. Suppose that there is a dense subset $D$ in $\mathscr{H}$ so that for $\varphi \in D$ the inequality (5) holds for some $p>1$. Then $H$ has purely absolutely continuous spectrum on $(a, b)$.

\section{Analytic dilation on complete manifolds with cor- ners of codimension 2}

In [Mü196] it has been explained how to meromorphically extend the resolvent of a generalized Laplacian on a complete manifold with corner of codimension 2, using the method outlined in section 1 under the hypothesis that the Laplacian on the corner has kernel $\{0\}$. It turns out that to weaken this hypothesis and try to use the methods of section 11 is not easy and new methods should be used to prove absence of singular continuous spectrum. In this section we survey the method of analytic dilation applied to compatible Laplacians on complete manifolds with corners of codimension 2. This method appeared originally in the context of Shrödinger operators and was adapted in Can11 to this geometric context.

Following Mül96, we explain the notions of compact and complete manifolds with corner of codimension 2. Let $X_{0}$ be a compact oriented Riemannian manifold with boundary $M$ and suppose that there exists a hypersurface $Y$ of $M$ that divides $M$ in two manifolds with boundary $M_{1}$ and $M_{2}$, i.e. $M=M_{1} \cup M_{2}$ and $Y=M_{1} \cap M_{2}$. Assume also that a neighborhood of $Y$ in $M$ is diffeomorphic to $Y \times(-\varepsilon, \varepsilon)$. We say that the manifold $X_{0}$ has a corner of codimension 2 if $X_{0}$ is endowed with a Riemannian metric $g$ that is a product metric on small neighborhoods, $M_{i} \times(-\varepsilon, 0]$ of the 
$M_{i}$ 's and on a small neighborhood, $Y \times(-\varepsilon, 0]^{2}$, of the corner $Y$. If $X_{0}$ has a corner of codimension 2 , we say that $X_{0}$ is a compact manifold with corner of codimension 2 .

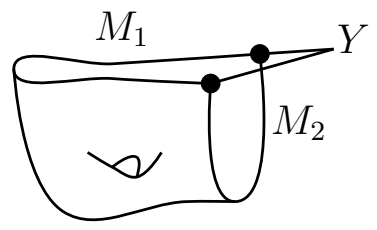

Figure 1. Compact manifold with corner of codimension 2.

Example 1 For $i=1,2$, let $M_{i}$ be a compact oriented Riemannian manifold with boundary $\partial M_{i}:=Y_{i}$. Suppose that on a neighborhood $Y_{i} \times(-\varepsilon, 0]$ of $Y_{i}$ the Riemannian metric $g_{i}$ of $M_{i}$ is a product metric i.e. $g_{i}:=g_{Y_{i}}+d u \otimes d u$ where $u$ is the coordinate associated to $(-\varepsilon, 0]$ in $Y_{i} \times(-\varepsilon, 0]$. Then $M_{1} \times M_{2}$ is a compact manifold with corner of codimension 2.

From the compact manifold with corner $X_{0}$ we construct a complete manifold $X$. Let

$$
Z_{i}:=M_{i} \cup_{Y}\left(\mathbb{R}^{+} \times Y\right), \mathrm{i}=1,2,
$$

where the bottom $\{0\} \times Y$ of the half-cylinder is identified with $\partial M_{i}=Y$. Then $Z_{i}$ is a manifold with cylindrical end (see definition 1). Define the manifolds

$$
W_{1}:=X_{0} \cup_{M_{2}}\left(\mathbb{R}_{+} \times M_{2}\right) \text { and } W_{2}:=X_{0} \cup_{M_{1}}\left(\mathbb{R}_{+} \times M_{1}\right) .
$$

Observe that $W_{i}$ is an $n$-dimensional manifold with boundary $Z_{i}$ that can be equipped with a Riemannian metric compatible with the product metric of $\mathbb{R}_{+} \times M_{2}$ and the Riemannian metric of $X_{0}$. Set:

$$
X:=W_{1} \cup_{Z_{1}}\left(\mathbb{R}_{+} \times Z_{1}\right)=W_{2} \cup_{Z_{2}}\left(\mathbb{R}_{+} \times Z_{2}\right),
$$

where we identify $\{0\} \times Z_{i}$ with $Z_{i}$, the boundary of $W_{i}$. 


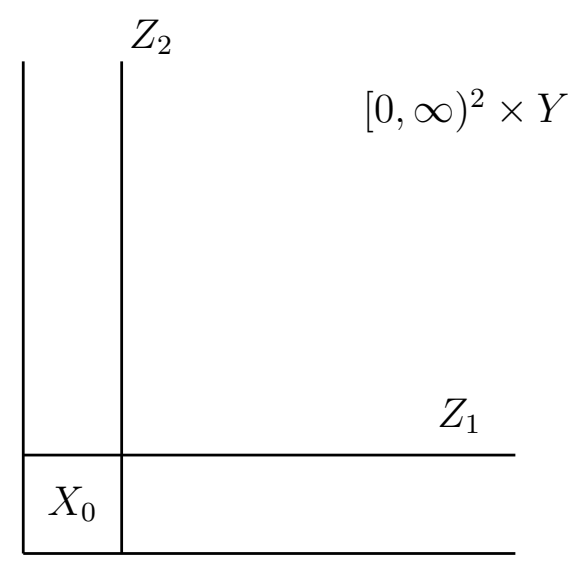

Figure 2. Sketch of a complete manifold with corner of codimension 2.

The above picture is an sketch, in particular the lines that enclosed the picture should not be thought as boundaries.

Let $T \geq 0$ be given and set

$$
Z_{i, T}:=M_{i} \cup_{Y}([0, T] \times Y), \text { for } i=1,2,
$$

where $\{0\} \times Y$ is identified with $Y$, the boundary of $M_{i} . Z_{i, T}$ is a family of manifolds with boundary which exhausts $Z_{i}$. Next we attach to $X_{0}$ the manifold $[0, T] \times M_{1}$ by identifying $\{0\} \times M_{1}$ with $M_{1}$. The resulting manifold $W_{2, T}$ is compact manifold with corner of codimension 2, whose boundary is the union of $M_{1}$ and $Z_{2, T}$. The manifold $X$ has associated a natural exhaustion given by

$$
X_{T}:=W_{2, T} \cup_{Z_{2, T}}\left([0, T] \times Z_{2, T}\right), T \geq 0
$$

where we identify $Z_{2, T}$ with $\{0\} \times Z_{2, T}$.

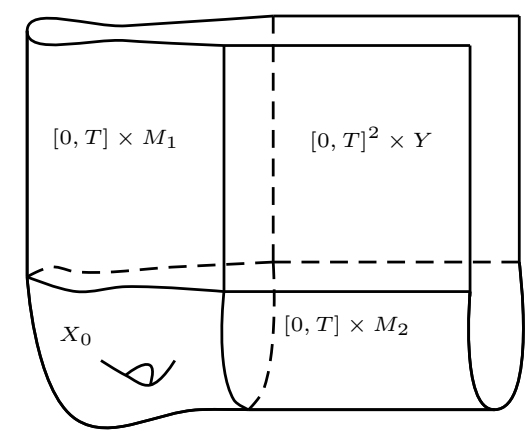

Figure 2. $X_{T}$, element of the exhaustion of $X$.

For each $T \in[0, \infty), X$ has two submanifolds with cylindrical ends, namely $M_{i} \times\{T\} \cup$ $(Y \times\{T\}) \times[0, \infty)$, for $i=1,2$. 
Let $E$ be a Hermitian vector bundle over a complete manifold with corner of codimension $2, X$. Let $\Delta$ be a generalized Laplacian acting on $C^{\infty}(X, E)$. The operator $\Delta$ is a compatible Laplacian over $X$ if it satisfies the following properties:

i) There exists a Hermitian vector bundle $E_{i}$ over $Z_{i}$ such that $\left.E\right|_{\mathbb{R}_{+} \times Z_{i}}$ is the pullback of $E_{i}$ under the projection $\pi: \mathbb{R}_{+} \times Z_{i} \rightarrow Z_{i}$, for $i=1$, 2. We suppose also that the Hermitian metric of $E$ is the pullback of the Hermitian metric of $E_{i}$. On $\mathbb{R}_{+} \times Z_{i}$, we have

$$
\Delta=-\frac{\partial^{2}}{\partial u_{i}^{2}}+\Delta_{Z_{i}}
$$

where $\Delta_{Z_{i}}$ is a compatible Laplacian acting on $C^{\infty}\left(Z_{i}, E_{i}\right)$.

ii) There exists a Hermitian vector bundle $S$ over $Y$ such that $\left.E\right|_{\mathbb{R}_{+}^{2} \times Y}$ is the pullback of $S$ under the projection $\pi: \mathbb{R}_{+}^{2} \times Y \rightarrow Y$. We assume also that the Hermitian product on $\left.E\right|_{\mathbb{R}_{+}^{2} \times Y}$ is the pullback of the Hermitian product on $S$. Finally we suppose that the operator $\Delta$ restricted to $\mathbb{R}_{+}^{2} \times Y$ satisfies

$$
\Delta=-\frac{\partial^{2}}{\partial u_{1}^{2}}-\frac{\partial^{2}}{\partial u_{2}^{2}}+\Delta_{Y},
$$

where $\Delta_{Y}$ is a generalized Laplacian acting on $C^{\infty}(Y, S)$.

Examples of compatible Laplacians are the Laplacian acting on forms and Laplacians associated to compatible Dirac operators (see [Mül96]). Since $X$ is a manifold with bounded geometry and the vector bundle $E$ has bounded Hermitian metric, the operator $\Delta: C_{c}^{\infty}(X, E) \rightarrow L^{2}(X, E)$ is essentially self-adjoint (see [Shu91, corollary 4.2]). Similarly $\Delta_{Z_{i}}: C_{c}^{\infty}\left(Z_{i}, E_{i}\right) \rightarrow L^{2}\left(Z_{i}, E_{i}\right)$ is also essentially self-adjoint for $i=1,2$.

Definition 2 - Let $H$ and $H^{(i)}$ be the self-adjoint extensions of $\Delta: C_{c}^{\infty}(X, E) \rightarrow$ $L^{2}(X, E)$ and $\Delta_{Z_{i}}: C_{c}^{\infty}\left(Z_{i}, E_{i}\right) \rightarrow L^{2}\left(Z_{i}, E_{i}\right)$.

- Let $b_{i}$ be the self-adjoint extension of $-\frac{d^{2}}{d u_{i}^{2}}: C_{c}^{\infty}\left(\mathbb{R}_{+}\right) \rightarrow L^{2}\left(\mathbb{R}_{+}\right)$obtained by imposing Dirichlet boundary conditions at 0 .

- Let $H_{i}$ be the self-adjoint operator $b_{i} \otimes I d+I d \otimes H^{(i)}$ acting on $L^{2}\left(\mathbb{R}_{+}\right) \otimes L^{2}\left(Z_{i}, E_{i}\right)$.

- Let $H^{(3)}$ be the self-adjoint operator associated to the essentially self-adjoint operator $\Delta_{Y}: C^{\infty}(Y, S) \rightarrow L^{2}(Y, S)$ and let $H_{3}$ be the self-adjoint operator $H_{3}:=$ $b_{1} \otimes I d \otimes I d+I d \otimes b_{2} \otimes I d+I d \otimes I d \otimes H^{(3)}$ acting on $L^{2}\left(\mathbb{R}_{+}\right) \otimes L^{2}\left(\mathbb{R}_{+}\right) \otimes L^{2}(Y)$.

- The operators $H_{i}$ are called channel operators for $i=1,2,3$. 
The self-adjoint operators $H_{1}$ and $H_{2}$ have a free channel of dimension 1 (associated to $b_{1}$ and $b_{2}$, respectively); the operator $H_{3}$ has a free channel of dimension 2 (associated to $\left.b_{1} \otimes I d \otimes I d+I d \otimes b_{2} \otimes I d\right)$. In some parts of this text we abuse the notation and denote by $H, H_{i}$, and $H^{(i)}$ the Laplacians acting on distributions and the self-adjoint operators previously defined.

Along the next section we will use the following notation. Let $H$ be a self-adjoint operator acting on a Hilbert space $\mathscr{H}$. We define the Banach space $\mathscr{H}_{2}(H)$ as the domain of $H$ with the norm $\|\varphi\|_{2}:=\|(|H|+i) \varphi\|$. Similarly, we define the Banach spaces $\mathscr{H}_{1}(H)$ as the completion of $\mathscr{H}_{2}(H)$ with the norm $\|\varphi\|_{1}:=\left\|(|H|+i)^{1 / 2} \varphi\right\|$, and $\mathscr{H}_{-1}(H)$ and $\mathscr{H}_{-2}(H)$ the dual spaces associated to $\mathscr{H}_{1}(H)$ and $\mathscr{H}_{2}(H)$.

\subsection{Analytic dilation}

The analytic dilation of a many-body Schrödinger operator depends on the analytic dilation of their subsystem Hamiltonians (see [?]). In a similar way the analytic dilation of $H$ is described in terms of the spectral theory of the operators $H^{(1)}, H^{(2)}$ and $H^{(3)}$, explained above. For $\theta>0$, we define the operator $U_{i, \theta}: L^{2}\left(Z_{i}\right) \rightarrow L^{2}\left(Z_{i}\right)$ that essentially is the dilation operator by $\theta+1$ up to a compact set. More precisely:

$$
U_{i, \theta} f(x)=\left\{\begin{array}{lc}
f(x) & \text { for } x \in M_{i} . \\
(\theta+1)^{1 / 2} f((\theta+1) u, y) & \text { for } x=(u, y) \in[0, \infty) \times Y \\
& \text { and for } u \text { big enough, }
\end{array}\right.
$$

and $U_{i, \theta} f$ is extended to the whole manifold $Z_{i}$ in such a way that it sends $C_{c}^{\infty}\left(Z_{i}\right)$ into $C_{c}^{\infty}\left(Z_{i}\right)$, and it becomes a unitary operator on $L^{2}\left(Z_{i}\right)$. We refer to Can11 for the technical details. Similarly, the operators $U_{\theta}: L^{2}(X) \rightarrow L^{2}(X)$ are defined by

$$
U_{\theta} f(x)=\left\{\begin{array}{lc}
f(x) & \text { for } x \in X_{0} . \\
(\theta+1)^{1 / 2} U_{i, \theta} f\left((\theta+1) u_{i}, z_{i}\right) & \text { for } x=\left(u_{i}, z_{i}\right) \in[0, \infty) \times Z_{i} \\
& \text { and for } u_{i} \text { big enough. }
\end{array}\right.
$$

Again $U_{\theta} f$ is extended to the whole $X$ in such a way that, for $f \in C_{c}^{\infty}(X), U_{\theta} f \in$ $C_{c}^{\infty}(X)$, and $U_{\theta}$ becomes a unitary operator in $L^{2}(X)$.

For $\theta \in[0, \infty)$, define $H_{\theta}:=U_{\theta} H U_{\theta}^{-1}$, a closed operator with domain $\mathscr{H}_{2}(H)$. We 
have that

$$
\mathscr{H}_{2}(H)=\left\{f \in L^{2}(X): \Delta_{\text {dist }} f \in L^{2}(X)\right\}
$$

is the second Sobolev space associated to $(X, g)$. Consider the set:

$$
\Gamma:=\left\{\theta:=\theta_{0}+i \theta_{1} \in \mathbb{C}: \theta_{0}>0, \theta_{0}>\left|\theta_{1}\right| \text { and } \operatorname{Im}(\theta)^{2}<1 / 2\right\}
$$

We will extend the family $H_{\theta}$ from $[0, \infty)$ to $\Gamma$.

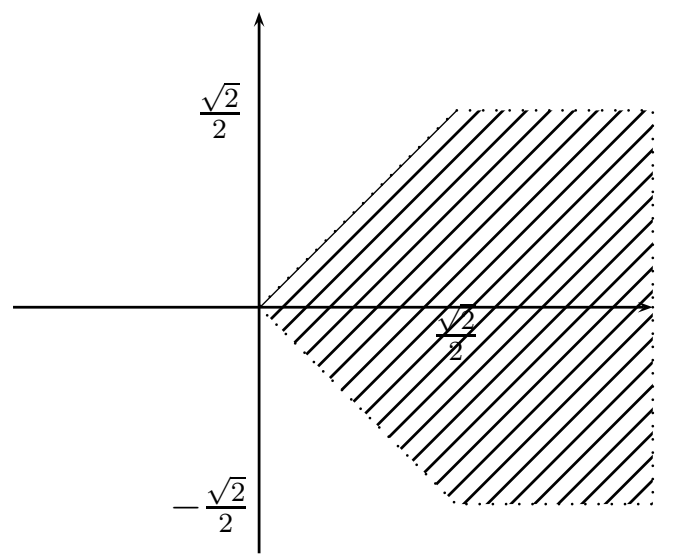

Figure 4. Sketch of the region $\Gamma$.

In [Can11], the next theorem is proved:

Theorem 5 [Can11] The family $\left(H_{\theta}\right)_{\theta \in[0, \infty)}$ extends to an holomorphic family for $\theta \in$ $\Gamma$, which satisfies:

1) $H_{\theta}$ is a closed operator with domain $\mathscr{H}_{2}(H)$ for $\theta \in \Gamma$.

2) For $\varphi \in \mathscr{H}_{2}(H)$ the map $\theta \mapsto H_{\theta} \varphi$ is holomorphic in $\Gamma$.

An holomorphic family of operators satisfying 1) and 2) is called a holomorphic family of type $\mathbf{A}$. This theorem is proved using the analogous result that the family $\left\{H^{(i), \theta}\right\}_{\theta \in[0, \infty)}$ extends to a holomorphic family of type $\mathrm{A}$ in $\Gamma$, where $H^{(i), \theta}$ denotes the closed operator associated to $U_{i, \theta} \Delta_{Z_{i}} U_{i, \theta}^{-1}$ with domain

$$
\mathscr{H}_{2}\left(H^{i}\right)=\left\{f \in L^{2}\left(Z_{i}\right): \Delta_{\text {dist }}(f) \in L^{2}\left(Z_{i}\right)\right\},
$$

the second Sobolev space associated to $\left(Z_{i}, g_{i}\right)$.

The families $H_{\theta}$ and $H_{\theta}^{(i)}$ extend to domains larger than $\Gamma$, but $\Gamma$ is enough for our goals. In particular, $\Gamma$ is chose because for $\theta \in \Gamma$ is easy to prove that $H_{\theta}^{(i)}$ is $m$-sectorial 
(see [Can11, section 2.7]), fact that will be important for the proof of theorem [6] where Ichinose lemma is a main tool. We define

$$
\theta^{\prime}:=\frac{1}{(\theta+1)^{2}}
$$

The parameter $\theta^{\prime}$ is very important in the description of the essential spectrum of $H_{\theta}$ as we can see in the next theorem.

Theorem 6 Can11] For $\theta \in \Gamma$,

$$
\begin{aligned}
\sigma_{e s s}\left(H_{\theta}\right)= & \bigcup_{\mu \in \sigma\left(H^{(3)}\right)}\left(\mu+\theta^{\prime}[0, \infty)\right) \\
& \cup \bigcup_{\lambda_{1} \in \sigma_{p p}\left(H^{(1), \theta}\right)}\left(\lambda_{1}+\theta^{\prime}[0, \infty)\right) \\
& \cup \bigcup_{\lambda_{2} \in \sigma_{p p}\left(H^{(2, \theta)}\right)}\left(\lambda_{2}+\theta^{\prime}[0, \infty)\right) .
\end{aligned}
$$

It is possible to associate to $\left(U_{\theta}\right)_{\theta \in[0, \infty)}$ a set of function $\mathscr{V} \subset \mathscr{H}_{2}(H)$ that satisfies:

i) $\mathscr{V}$ is dense in $L^{2}(X)$.

ii) for $\varphi \in \mathscr{V}, U_{\theta} \varphi$ is defined for all $\theta \in \Gamma$.

iii) $U_{\theta} \mathscr{V}$ is dense in $L^{2}(X)$ for all $\theta \in \Gamma$.

The elements of a subset of $\mathscr{H}_{2}(H)$ which satisfies i) and ii) will be called analytic vectors. We denote by $\Lambda$ the left-hand plane, more explicitly:

$$
\Lambda:=\{(x, y) \in \mathbb{C}: x<0\}
$$

We denote by $R(\lambda)$ the resolvent of $H$ and by $R(\lambda, \theta)$ the resolvent of $H_{\theta}$. Using the general analytic dilation theory of Aguilar-Balslev-Combes (see [Bal97]) we describe the nature of the spectrum of $H$. This theory is based on:

i) The knowledge of the essential spectrum of $H_{\theta}$, provided by theorem 6 ,

ii) The following equation, that is consequence of the unitarity of $U_{\theta}$,

$$
\langle R(\lambda) f, g\rangle_{L^{2}(X)}=\left\langle R(\lambda, \theta) U_{\theta} f, U_{\theta} g\right\rangle_{L^{2}(X)},
$$

for $f, g \in \mathscr{V}$ and $\theta \in[0, \infty)$. 
Since the right-hand side of (10) is defined for $\lambda \in \Lambda$ and $\theta \in \Gamma$, (10) provides a meromorphic extension of the functions $\lambda \mapsto\langle R(\lambda) f, g\rangle_{L^{2}(X)}$ from $\Lambda$ to $\mathbb{C}-\sigma\left(H_{\theta}\right)$. From this, using Aguilar-Balslev-Combes, we deduce the following theorem.

Theorem 7 [Can11] 1) For $f, g \in \mathscr{A}$ the function $\lambda \mapsto\langle R(\lambda) f, g\rangle_{L^{2}(X)}$ extends from $\Lambda$ to $\mathbb{C}-\sigma\left(H_{\theta}\right)$.

2) For all $\theta \in \Gamma, H_{\theta}$ has no singular continuous spectrum.

3) The accumulation points of $\sigma_{p p}(H)$ are contained in $\{\infty\} \cup \sigma\left(H^{(3)}\right) \cup \cup_{i=1}^{2} \sigma_{p p}\left(H^{(i)}\right)$.

In the case of manifolds with cylindrical ends we have shown in section 1 the absence of singular continuous spectrum for their Laplacians; in Don84, by giving a polynomial bound to the growing of the number of $L^{2}$-eigenvalues, it is proved that the unique possible accumulation point of the pure point spectrum of a Laplacian on a manifold with cylindrical end is $\infty$.

\section{Acknowledgment}

The author is very grateful with the organizers of the summer school of Villa de Leyva 2011 for giving him the opportunity to participate in this nice school and to write this paper for its proceedings. Special thanks are due to Alexander Cardona who improves a lot the presentation of this paper. He wants to thanks also Professor Werner Müller at Bonn University for his constant advise that introduced him in the topics of this text and supported the writing of his PhD-thesis on which section 2 and [Can11] are based.

\section{References}

[Ba197] E. Balslev. Spectral deformation of Laplacians on hyperbolic manifolds. Comm. Anal. Geom., 5(2):213-247, 1997.

[Can11] L. Cano. Analytic dilation for Laplacians on manifolds with corners of codimension 2. arXiv:1103.0937v1. 2011.

[Cha84] I. Chavel. Eigenvalues in Riemannian geometry, volume 115 of Pure and Applied Mathematics. Academic Press Inc., Orlando, FL, 1984. Including a chapter by Burton Randol, With an appendix by Jozef Dodziuk. 
[DEM98] P. Duclos, P. Exner, and B. Meller. Exponential bounds on curvature-induced resonances in a two-dimensional Dirichlet tube. Helv. Phys. Acta, 71(2):133$162,1998$.

[Don84] H. Donnelly. Eigenvalue estimates for certain noncompact manifolds. Michigan Math. J., 31(3):349-357, 1984.

[Gér93] C. Gérard. Distortion analyticity for $N$-particle Hamiltonians. Helv. Phys. Acta, 66(2):216-225, 1993.

[Gui89] L. Guillopé. Théorie spectrale de quelques variétés à bouts. Ann. Sci. École Norm. Sup. (4), 22(1):137-160, 1989.

[Hus05] R. Husseini. Zur spektraltheorie verallgemeinerter laplace-operatoren auf mannigfaltigkeiten mit zylindrischen enden. Diplomarbeit, Rheinischen Friedrich-Wilhelms-Universität Bonn, 2005.

[Kal10] V. Kalvin. The aguilar-baslev-combes theorem for the laplacian on a manifold with an axial analytic asymptotically cylindrical end. Arxiv 10032538v2, 2010.

[KS07] H. Kovařík and A. Sacchetti. Resonances in twisted quantum waveguides. J. Phys. A, 40(29):8371-8384, 2007.

[Mül83] W. Müller. Spectral theory for Riemannian manifolds with cusps and a related trace formula. Math. Nachr., 111:197-288, 1983.

[Mül96] Werner Müller. On the $L^{2}$-index of Dirac operators on manifolds with corners of codimension two. I. J. Differential Geom., 44(1):97-177, 1996.

[MV02] R. Mazzeo and A. Vasy. Resolvents and Martin boundaries of product spaces. Geom. Funct. Anal., 12(5):1018-1079, 2002.

[MV04] R. Mazzeo and A. Vasy. Analytic continuation of the resolvent of the Laplacian on SL(3)/SO(3). Amer. J. Math., 126(4):821-844, 2004.

[MV07] R. Mazzeo and A. Vasy. Scattering theory on $\operatorname{SL}(3) / \mathrm{SO}(3)$ : connections with quantum 3-body scattering. Proc. Lond. Math. Soc. (3), 94(3):545-593, 2007.

[Ros97] Steven Rosenberg. The Laplacian on a Riemannian manifold, volume 31 of London Mathematical Society Student Texts. Cambridge University Press, Cambridge, 1997. An introduction to analysis on manifolds. 
[RS79] M. Reed and B. Simon. Methods of modern mathematical physics. III. Academic Press [Harcourt Brace Jovanovich Publishers], New York, 1979. Scattering theory.

[RS80] M. Reed and B. Simon. Methods of modern mathematical physics. I. Academic Press Inc. [Harcourt Brace Jovanovich Publishers], New York, second edition, 1980. Functional analysis.

[Shu91] M.A. Shubin. Spectral theory of elliptic operators on non-compact manifolds. Paper on lectures Summer School on Semiclassical Methods, Nantes, 1991.

[SZ93a] J. Sjöstrand and M. Zworski. Estimates on the number of scattering poles near the real axis for strictly convex obstacles. Ann. Inst. Fourier (Grenoble), 43(3):769-790, 1993.

[SZ93b] J. Sjöstrand and M. Zworski. Lower bounds on the number of scattering poles. Comm. Partial Differential Equations, 18(5-6):847-857, 1993.

[SZ94] J. Sjöstrand and M. Zworski. Lower bounds on the number of scattering poles. II. J. Funct. Anal., 123(2):336-367, 1994.

[SZ95] J. Sjöstrand and M. Zworski. The complex scaling method for scattering by strictly convex obstacles. Ark. Mat., 33(1):135-172, 1995. 